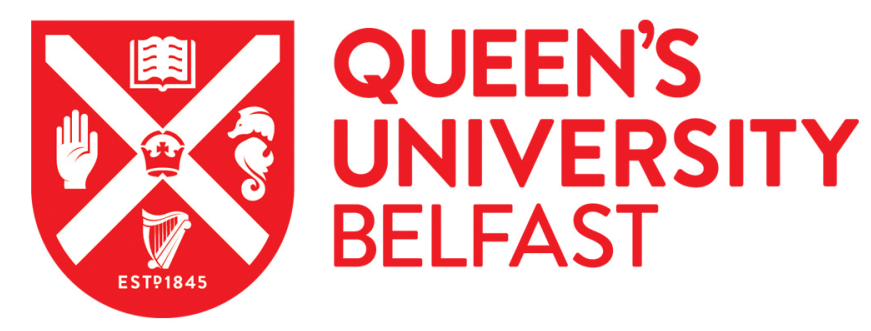

\title{
Generalised supersolutions with mass control for the Keller-Segel system with logarithmic sensitivity
}

Zhigun, A. (2018). Generalised supersolutions with mass control for the Keller-Segel system with logarithmic sensitivity. Journal of Mathematical Analysis and Applications, 467(2), 1270-1286.

https://doi.org/10.1016/j.jmaa.2018.08.001

Published in:

Journal of Mathematical Analysis and Applications

Document Version:

Peer reviewed version

Queen's University Belfast - Research Portal:

Link to publication record in Queen's University Belfast Research Portal

Publisher rights

(c) 2018 Elsevier B. V.

This manuscript version is made available under the CC-BY-NC-ND 4.0 license http://creativecommons.org/licenses/by-nc-nd/4.0/,which permits distribution and reproduction for non-commercial purposes, provided the author and source are cited.

\section{General rights}

Copyright for the publications made accessible via the Queen's University Belfast Research Portal is retained by the author(s) and / or other copyright owners and it is a condition of accessing these publications that users recognise and abide by the legal requirements associated with these rights.

Take down policy

The Research Portal is Queen's institutional repository that provides access to Queen's research output. Every effort has been made to ensure that content in the Research Portal does not infringe any person's rights, or applicable UK laws. If you discover content in the Research Portal that you believe breaches copyright or violates any law, please contact openaccess@qub.ac.uk. 


\title{
Generalised supersolutions with mass control for the Keller-Segel system with logarithmic sensitivity
}

\author{
Anna Zhigun \\ Technische Universität Kaiserslautern, Felix-Klein-Zentrum für Mathematik \\ Paul-Ehrlich-Str. 31, 67663 Kaiserslautern, Germany \\ e-mail: zhigun@mathematik.uni-kl.de
}

\begin{abstract}
The existence of generalised global supersolutions with a control upon the total muss is established for the parabolic-parabolic Keller-Segel system with logarithmic sensitivity for any space dimension. It is verified that smooth supersolutions of this sort are actually classical solutions. Unlike the previously existing constructions, neither is the chemotactic sensitivity coefficient required to be small, nor is it necessary for the initial data to be radially symmetric.
\end{abstract}

Keywords: chemotaxis; generalised supersolution; global existence; logarithmic sensitivity. MSC 2010: 35B45, 92C17, 35D30, 35D99, 35K55.

\section{Introduction}

Coupled reaction-diffusion-transport PDEs are a standard tool in the mathematical modelling of cell motility on the macroscale. Thereby, the diffusion-dominated systems are among the best studied analytically. Standard theory (see, e.g., [13]) ensures the existence of bounded solutions to such systems. Still, they are not always the optimal choice. Indeed, in many instances it is not the chaotic movement but, rather, the active drift of the cells towards some substance which actually dominates the motion and, as a result, may lead to a strong aggregation of the biomass density. The best known model example of such a situation is provided by the celebrated Keller-Segel system for chemotaxis [11, 12]. This parabolicparabolic system and its parabolic-elliptic simplifications have been objects of extensive studies in recent decades. It turned out that in higher spatial dimensions the solutions to such systems can exhibit a blowup in finite time which calls into question the global solvability. For certain parabolic-elliptic versions of the classical Keller-Segel model on the plane one was able to extend the solutions which collapse into a persistent Dirac-type singularity beyond a finite-time blow-up by constructing measure-valued continuations $[16,21]$. For a detailed overview of available results concerning boundedness/blow-up, as well as other properties, of the Keller-Segel model the reader is referred to [2, 10].

Very recently a new solution concept was introduced [15] in the context of a version of the Keller-Segel system with a signal-dependent chemotactic sensitivity:

$$
\begin{cases}\partial_{t} u=\nabla \cdot\left(\nabla u-\chi \frac{u}{v} \nabla v\right) & \text { in } \mathbb{R}^{+} \times \Omega, \\ \partial_{t} v=\Delta v-v+u & \text { in } \mathbb{R}^{+} \times \Omega, \\ \partial_{\nu} u=\partial_{\nu} v=0 & \text { in } \mathbb{R}^{+} \times \partial \Omega, \\ u(0, \cdot)=u_{0}, v(0, \cdot)=v_{0} & \text { in } \Omega,\end{cases}
$$

where $\Omega$ is a smooth bounded domain in $\mathbb{R}^{n}, n \in \mathbb{N}$, with the corresponding outer normal unit vector $\nu$ on $\partial \Omega$, and $\chi$ is a positive number. In this model prototype the cells are assumed to respond to the changes of the logarithm of the signal concentration thus following the Weber-Fechner law. Due to the saturation effect upon the chemotactic sensitivity in the presence of high levels of signal concentration, the solutions of both (1.1) and the corresponding parabolic-elliptic versions are less prone to the formation of strong singularities, such as, e.g., Dirac measures, than those of the classical Keller-Segel model. In particular, the global existence of bounded classical [2, 6-9, 14, 17, 18, 22, 25], weak [20, 22], and generalised [3, 15] solutions was established for certain ranges of parameter $\chi$ which depend upon $n$ and, also, on whether the setting is radial-symmetric or not. On the other hand, it is known, for a parabolic-elliptic case at 
least [18] that blow-up solutions exist for $n \geqslant 3$ and $\chi$ large enough. To the best of our knowledge, no solution concept has as yet been proposed for (1.1) which would allow to treat the general non-radial symmetric setting and arbitrary large $\chi>0$ if $n \geqslant 3$. The present work aims to fill this gap.

In [15], the authors introduced the coupled quantity

$$
F(u, v)=u^{p} v^{q}
$$

for

$$
p, q \in(0,1)
$$

and derived a variational inequality which it should satisfy provided that $u$ and $v$ solve (1.1) in the classical sense. They showed that such inequality, when complemented by a control upon the total mass and equation (1.1b) fulfilled in the usual weak sense, together comprise a reasonable concept of a global solution to the full parabolic-parabolic system (1.1). This construction made it possible to extend the range of $\chi$ 's for which (1.1) can be considered as globally solvable, namely:

$$
0<\chi< \begin{cases}\infty & \text { if } n=2 \\ \sqrt{8} & \text { if } n=3 \\ \frac{n}{n-2} & \text { if } n \geqslant 4\end{cases}
$$

Indeed, previously available results on global solvability presupposed either a more restrictive condition on $\chi$ [22], or the radial symmetry requirement [20]. The generalised solution from [15] is a limit of a regularising sequence. Thanks to condition (1.3), the $u$-component of this sequence is uniformly integrable over arbitrary finite time-space cylinders. An important consequence of the later is the fact that the generalised solution satisfies equation $(1.1 \mathrm{~b})$ in the usual weak sense. In this paper we further develop the framework presented in [15]. The key idea is to replace (1.2) with

$$
F(u, v)=u^{-a} v^{-b}
$$

where parameters $a$ and $b$ are assumed to satisfy

$$
a>0 \quad \text { and } \quad b>b_{+}(a):=\frac{1+a}{2}\left(\sqrt{1+\chi^{2} a}-1\right),
$$

so that clearly

$$
b>0
$$

as well. An advantage of switching to a negative power of $u$ in (1.4) is that the imposed restriction on $b$ in (1.5) can be met for any $\chi>0$. On the negative side is that the $u$-components of the approximation sequence need no longer be locally in time uniformly integrable. As a result, the limit pair $(u, v)$, while remaining a supersolution to (1.1b), may, however, fail to be its subsolution, even in the weak sense. Thus, our approach to (1.1b) is to replace it by an identity which contains a certain nonnegative Radon measure. Notwithstanding, thanks to a suitable mass control involving this measure and an accurate description of the boundary conditions based on the concept of normal traces for divergence-measure fields [5], our generalised supersolution coincides with a classical solution in the case of regular $u$ and $v$.

The rest of the paper is organised in the following way. In Section 2 we introduce and discuss the assumptions on the initial data, the proposed supersolution concept (cl. Definition 2.2), and two main results: Theorem 2.6 deals with existence of such supersolutions, while Theorem 2.7 establishes a connection to the classical solvability. In Section 3 we prepare some ingredients which are necessary to our proof of Theorem 2.6 in Section 4. Theorem 2.7 is proved in the closing Section 5. Finally, Appendix A contains some facts on the divergence-measure fields which we use in this paper.

Acknowledgment. The author expresses her thanks to the reviewers for their helpful comments.

\section{Generalised supersolutions to (1.1)}

We assume throughout that the initial data satisfies the following assumptions:

$$
0<u_{0}, v_{0} \in L^{1}(\Omega)
$$

and

$$
u_{0}^{-a} v_{0}^{-b} \in L^{1}(\Omega) \quad \text { for some } a, b>0
$$


Remark 2.1 (Initial conditions).

1. Conditions (2.1)-(2.2) are to be compared to (1.7) in [15]. We impose less regularity for $u_{0}$ and $v_{0}$, as well as allow, e.g., $v_{0}$ to touch zero at some points in $\bar{\Omega}$. Yet, since we deal with negative powers of $u_{0}$, we cannot consider general $u_{0} \geqslant 0$ which vanish on sets of non-zero Lebesgue measure.

2. In our existence result, Theorem 2.6 below, we require (1.5) to hold in addition. Observe that $b_{+}$ is a strictly increasing function, and one readily sees that

$$
b_{+}(a) \underset{a \rightarrow 0}{\rightarrow} 0 .
$$

Hence, given any small $b$, we only need to satisfy $a \in\left(0,\left(b_{+}\right)^{-1}(b)\right)$, so that $a$ can be chosen arbitrary small as well. On the whole, this makes it easier to satisfy (1.5).

Motivated by an idea from [15] we introduce the following concept of a generalised supersolution with a mass control:

Definition 2.2 (Generalised supersolution). Let $\left(u_{0}, v_{0}\right)$ satisfy conditions (2.1) and (2.2). We call a pair of measurable functions $(u, v): \mathbb{R}_{0}^{+} \times \bar{\Omega} \rightarrow \mathbb{R}^{+} \times \mathbb{R}^{+}$a generalised supersolution to system (1.1) if

(i) $u \in L^{\infty}\left(\mathbb{R}^{+} ; L^{1}(\Omega)\right), v \in L_{l o c}^{1}\left(\mathbb{R}_{0}^{+} ; W^{1,1}(\Omega)\right), v^{-1} \in L_{l o c}^{\infty}\left(\mathbb{R}^{+} \times \bar{\Omega}\right)$;

(ii) $u^{-\frac{a}{2}} v^{-\frac{b}{2}} \in L_{l o c}^{2}\left(\mathbb{R}_{0}^{+} ; H^{1}(\Omega)\right) \cap L_{l o c}^{\infty}\left(\mathbb{R}_{0}^{+} ; L^{2}(\Omega)\right), u^{-\frac{a}{2}} v^{-\frac{b}{2}-1} \nabla v \in L_{l o c}^{2}\left(\mathbb{R}_{0}^{+} ; L^{2}(\Omega)\right)$, $u^{-a+1} v^{-b-1} \in L_{l o c}^{1}\left(\mathbb{R}_{0}^{+} ; L^{1}(\Omega)\right)$;

(iii) $\int_{0}^{\infty} \psi\left(\nabla\left(u^{-a} v^{-b}\right)+\chi a u^{-a} v^{-b-1} \nabla v\right) d s, \int_{0}^{\infty} \psi \nabla v d s \in \mathcal{D} \mathcal{M}^{p}(\Omega)$ for all $\psi \in C_{0}^{1}\left(\mathbb{R}_{0}^{+}\right)$for some $p \in$ $\left(1, \frac{n}{n-1}\right)$;

(iv) for all $0 \leqslant \varphi \in C^{1}(\bar{\Omega})$ and $0 \leqslant \psi \in C_{0}^{1}\left(\mathbb{R}_{0}^{+}\right)$it holds that

$$
\begin{aligned}
& \quad-\int_{0}^{\infty} \partial_{t} \psi \int_{\Omega} u^{-a} v^{-b} \varphi d x d t-\psi(0) \int_{\Omega} u_{0}^{-a} v_{0}^{-b} \varphi d x \\
& \leqslant-4 \int_{0}^{\infty} \psi \int_{\Omega}\left(\frac{a+1}{a}\left|\nabla\left(u^{-\frac{a}{2}} v^{-\frac{b}{2}}\right)\right|^{2}+\left(\frac{b}{a}+\chi \frac{a+1}{2}\right) \nabla\left(u^{-\frac{a}{2}} v^{-\frac{b}{2}}\right) \cdot u^{-\frac{a}{2}} v^{-\frac{b}{2}-1} \nabla v\right. \\
& \left.\quad+\frac{1}{4}\left(\frac{b^{2}}{a}+b+\chi b\right)\left|u^{-\frac{a}{2}} v^{-\frac{b}{2}-1} \nabla v\right|^{2}\right) \varphi d x d s \\
& -\int_{0}^{\infty} \psi \int_{\Omega}\left(\nabla\left(u^{-a} v^{-b}\right)+\chi a u^{-a} v^{-b-1} \nabla v\right) \cdot \nabla \varphi d x d s \\
& +\int_{0}^{\infty} \psi \int_{\Omega}\left(b u^{-a} v^{-b}-b u^{-a+1} v^{-b-1}\right) \varphi d x d s
\end{aligned}
$$

(v) there exists some non-negative Radon measure $\mu$ in $\mathbb{R}_{0}^{+} \times \bar{\Omega}$, s.t. for all $0 \leqslant \varphi \in C^{1}(\bar{\Omega})$ and $0 \leqslant \psi \in C_{0}^{1}\left(\mathbb{R}_{0}^{+}\right)$it holds that

$$
-\int_{0}^{\infty} \partial_{t} \psi \int_{\Omega} v \varphi d x d s-\psi(0) \int_{\Omega} v_{0} \varphi d x=\int_{0}^{\infty} \psi \int_{\Omega}-\nabla v \cdot \nabla \varphi+(-v+u) \varphi d x d s+\int_{0}^{\infty} \psi \int_{\bar{\Omega}} \varphi d \mu(s, x),
$$

and

$$
\int_{0}^{\infty} \psi\|u(t, \cdot)\|_{L^{1}(\Omega)} d s+\int_{0}^{\infty} \psi \int_{\bar{\Omega}} d \mu(s, x) \leqslant\left\|u_{0}\right\|_{L^{1}(\Omega)}\|\psi\|_{L^{1}\left(\mathbb{R}^{+}\right)}
$$

(vi) for all $\psi \in C_{0}^{1}\left(\mathbb{R}_{0}^{+}\right)$it holds that

$$
\begin{array}{ll}
\left.\int_{0}^{\infty} \psi\left(\nabla\left(u^{-a} v^{-b}\right)+\chi a u^{-a} v^{-b-1} \nabla v\right) d s \cdot \nu\right|_{\partial \Omega}=0 & \text { in } W^{-\frac{1}{p}, p}(\partial \Omega), \\
\left.\int_{0}^{\infty} \psi \nabla v d s \cdot \nu\right|_{\partial \Omega}=0 & \text { in } W^{-\frac{1}{p}, p}(\partial \Omega) .
\end{array}
$$

Several remarks are in order. 
Remark 2.3 (Boundary conditions). The variational reformulations (2.6)-(2.7) of the boundary conditions (1.1c) are consistent with the regularity assumptions in (iii), cl. [5, Theorem 2.1]. Some necessary facts on the spaces $\mathcal{D M}^{p}(\Omega)$ are recalled for the reader's convenience in Appendix A.

Remark 2.4 (Coupled quantities).

1. One of the well-known difficulties to be faced while dealing with a system like (1.1) in higher dimensions is the generally poor regularity of variable $u$. The reason lies in a comparative weakness of linear diffusion which often fails to compensate the aggregation due to taxis. At the same time, variable $v$ is much better-behaved. Our previous studies of highly-degenerate haptotaxis systems $[26,27]$ suggest that it can be helpful to introduce a quantity which involves both variables and possesses an integrable gradient. Thereby, the choice of such coupled quantity depends to a large extent upon estimates one is able to derive for a given system. In particular, in the present case it turns out to be fruitful to study the term $u^{-\frac{a}{2}} v^{-\frac{b}{2}}$ and to include its gradient into the supersolution concept.

2. Since $u^{-\frac{a}{2}} v^{-\frac{b}{2}} \in L_{l o c}^{2}\left(\mathbb{R}_{0}^{+} ; H^{1}(\Omega)\right)$, we have due to the weak chain rule and the Hölder inequality that $u^{-\frac{a}{2}} v^{-\frac{b}{2}} \in L_{l o c}^{1}\left(\mathbb{R}_{0}^{+} ; W^{1,1}(\Omega)\right)$. On the whole, the regularity imposed by (i)-(iii) ensures that all integrals in the variational formulations (2.3)-(2.7) do make sense.

Remark 2.5 (Comparison with the solution concept from [15]). The main difference between our construction and the generalised solution concept in [15] is the presence of a nonnegative and, in general, nonzero measure $\mu$ on the right-hand side in the weak formulation (2.4). This means that the pair $(u, v)$ is actually a weak supersolution of $(1.1 \mathrm{~b})$ but may, however, fail to be its subsolution. In particular, even if both $u$ and $v$ are smooth functions, the variational properties (2.3)-(2.4) do not on their own imply that $(u, v)$ is a supersolution to (2.3). Fortunately, as it turns out, this property can be saved by taking into account the boundary conditions in form of (2.6)-(2.7), see the proof of Theorem 2.7 below.

A control from above upon the total mass of the $u$-component of a generalised supersolution to system (1.1) was used in [15], as well as, for some similar chemotaxis problems, in [23, 24]. There it took the basic form

$$
\|u(t, \cdot)\|_{L^{1}(\Omega)} \leqslant\left\|u_{0}\right\|_{L^{1}(\Omega)} \quad \text { for all } t \geqslant 0 .
$$

In our case (2.8) is replaced by a more general condition (2.5) which involves the aforementioned measure $\mu$.

Our result on existence now reads:

Theorem 2.6 (Existence of generalised supersolutions). Let $\chi$ be any positive number. Let the initial conditions $u_{0}$ and $v_{0}$ satisfy (2.1)-(2.2) for some constants $a$ and $b$ which fulfil (1.5). Then there exists a generalised supersolution $(u, v)$ in terms of Definition 2.2.

The proof of this theorem is based on a suitable regularisation and a series of priori estimates in Section 3 leading into a limit procedure in Section 4.

Our interest in the introduced concept of generalised supersolutions is supported by the following result:

Theorem 2.7 (Classical solutions). Let a pair $(u, v)$ be a supersolution in terms of Definition 2.2. Assume in addition that

$$
u, v \in C^{1,2}\left(\mathbb{R}_{0}^{+} \times \bar{\Omega}\right)
$$

and

$$
\frac{u}{v} \in L_{l o c}^{\infty}\left(\mathbb{R}_{0}^{+} \times \bar{\Omega}\right)
$$

Then $(u, v)$ solves (1.1) in the classical sense.

The proof of Theorem 2.7 is given in Section 5.

Remark 2.8 (Multiple time scales). Should $u$ and $v$ evolve on different time scales, one could account for this by introducing a scaling parameter $0<\tau \neq 1$ into equation (1.1a):

$$
\tau \partial_{t} u=\nabla \cdot\left(\nabla u-\chi \frac{u}{v} \nabla v\right)
$$


Our results and, moreover, also their proofs, with obvious minor alterations, continue to hold in this case as well. The only substantial change regards the restrictions on $a$ and $b$ : assumption (1.5) has to be replaced by

$$
a, b>0 \quad \text { and } \quad\left((1-\tau)^{2} a-4 \tau\right) b^{2}+2((1-\tau) \chi a-2 \tau) b(a+1)+\chi^{2} a(a+1)^{2}<0 .
$$

Obviously, (2.10) can be met for any $\chi>0$ and $0<\tau \neq 1$ by fixing some

$$
0<a<a_{+}(\tau):=\frac{4 \tau}{(1-\tau)^{2}},
$$

and then choosing $b$ sufficiently large. In particular, no smallness assumption on $\tau$ needs to be imposed, as was the done, e.g., in [7]. Altogether, this is a further confirmation of the robustness of the concept of the generalised solvability as proposed in Definition 2.2 if compared to the classical one.

Remark 2.9 (Notation). We make the following useful convention: For any index $i$, a quantity $C_{i}$ denotes a positive constant or, alternatively, a positive function of its arguments. Moreover, dependence upon such parameters as: the space dimension $n$, domain $\Omega$, constants $a, b, \chi$, as well as the structure of the initial data $u_{0}, v_{0}$, is mostly not indicated in an explicit way.

\section{Smooth regularisations for (1.1)}

Let

$$
2 \leqslant k \in \mathbb{N}
$$

Following [15], we consider a family of regularisations of system (1.1):

$$
\begin{cases}\partial_{t} u_{k}=\nabla \cdot\left(\nabla u_{k}-\chi \frac{u_{k}}{v_{k}} \nabla v_{k}\right) & \text { in }(0, T) \times \Omega, \\ \partial_{t} v_{k}=\Delta v_{k}-v_{k}+\frac{u_{k}}{1+\frac{1}{k} u_{k}} & \text { in }(0, T) \times \Omega, \\ \partial_{\nu} u_{k}=\partial_{\nu} v_{k}=0 & \text { in }(0, T) \times \partial \Omega, \\ u_{k}(0, \cdot)=u_{k 0}, v_{k}(0, \cdot)=v_{k 0} & \text { in } \Omega .\end{cases}
$$

Thereby, we choose the regularised initial data $u_{k 0}$ and $v_{k 0}$ so as to satisfy

$$
u_{k 0}, v_{k 0} \in W^{1, \infty}(\Omega), \quad \min _{\bar{\Omega}} u_{k 0}, \min _{\bar{\Omega}} v_{k 0}>0
$$

as well as to be suitable approximations to the original starting values, $u_{0}$ and $v_{0}$, see next sequel. Classical theory for upper-triangular systems (see, e.g., [1]) implies that (3.1) possesses a unique global classical solution $\left(u_{k}, v_{k}\right)$. Moreover, due to the maximum principle, both solution components are strictly positive in $\mathbb{R}_{0}^{+} \times \bar{\Omega}$. These solutions are studied in Subsections 3.2-3.4.

\subsection{Approximation of initial data}

Apart from being smooth, we assume that $u_{k 0}$ and $v_{k 0}$ fulfil the following conditions: for all $k \in \mathbb{N}$ it holds that

$$
\begin{array}{ll}
u_{k 0} \underset{k \rightarrow \infty}{\rightarrow} u_{0} & \text { in } L^{1}(\Omega) \text { and a.e. in } \Omega, \\
v_{k 0} \underset{k \rightarrow \infty}{\rightarrow} v_{0} & \text { in } L^{1}(\Omega) \text { and a.e. in } \Omega, \\
u_{k 0}^{-a} v_{k 0}^{-b} \underset{k \rightarrow \infty}{\rightarrow} u_{0}^{-a} v_{0}^{-b} & \text { in } L^{1}(\Omega) \text { and a.e. in } \Omega .
\end{array}
$$

Let us check that these conditions can be met. First, we observe that since $u_{0}, v_{0} \in L^{1}(\Omega)$, there exist some sequences $u_{k 0}$ and $v_{k 0}$ which satisfy (3.2) and (3.3)-(3.4), as well as

$$
u_{k 0} \geqslant \begin{cases}\max \left\{k^{-\frac{b}{a}}, u_{0}\right\} & \text { for } u_{0}<k \\ k & \text { for } u_{0} \geqslant k\end{cases}
$$




$$
v_{k 0} \geqslant \begin{cases}\max \left\{k^{-\frac{a}{b}}, v_{0}\right\} & \text { for } u_{0}<k, \\ k & \text { for } v_{0} \geqslant k .\end{cases}
$$

Using (3.6)-(3.7), we compute that

$$
u_{k 0}^{-a} v_{k 0}^{-b} \leqslant \max \left\{u_{0}^{-a} v_{0}^{-b}, 1\right\} \quad \text { a.e. in } \Omega \text {. }
$$

Combining (2.2), (3.3)-(3.4), and (3.8) with the dominated convergence theorem we obtain (3.5).

\subsection{Basic properties of (3.1)}

Integrating equations (3.1a) and (3.1b) over $\Omega$ and using the boundary conditions and partial integration we obtain the following information about the total masses: for all $t \geqslant 0$

$$
\begin{aligned}
\left\|u_{k}(t, \cdot)\right\|_{L^{1}(\Omega)} & =\left\|u_{k 0}\right\|_{L^{1}(\Omega)}, \\
\left\|v_{k}(t, \cdot)\right\|_{L^{1}(\Omega)} & \leqslant\left(1-e^{-t}\right)\left\|u_{k 0}\right\|_{L^{1}(\Omega)}+e^{-t}\left\|v_{k 0}\right\|_{L^{1}(\Omega)} .
\end{aligned}
$$

Due to (3.9)-(3.10) and a classical result based on duality (see, e.g., the proof of Lemma 5 in [4, Appendix A]) we have for all

$$
(r, s) \in\left[1, \frac{n+2}{n}\right) \times\left[1, \frac{n+2}{n+1}\right)
$$

that

$$
\left\{\left(v_{k}, \nabla v_{k}\right)\right\}_{k \in(0,1]} \quad \text { is precompact in } L^{r}((0, T) \times \Omega) \times L^{s}((0, T) \times \Omega) .
$$

Further, using the maximum principle and the strict positivity of the Neumann heat kernel, we conclude from $(3.1 \mathrm{~b})$ and (3.4) that $v_{k}$ can be controlled from below in the following way:

$$
\begin{aligned}
\inf _{(\tau, T) \times \Omega} v_{k} & \geqslant \inf _{(\tau, T) \times \Omega} e^{-t} e^{t \Delta} v_{k 0} \\
& \geqslant C_{1}(\tau, T)\left\|v_{k 0}\right\|_{L^{1}(\Omega)} \\
& \geqslant C_{2}(\tau, T)>0 \quad \text { for all } 0<\tau<T<\infty .
\end{aligned}
$$

\subsection{A variational formulation for (3.1)}

Let $F \in C^{2}\left(\mathbb{R}^{+} \times \mathbb{R}^{+}\right)$. Multiplying (3.1a) and (3.1b) by $\partial_{u} F\left(u_{k}, v_{k}\right)$ and $\partial_{v} F\left(u_{k}, v_{k}\right)$, respectively, adding the results together, and using the chain rule where necessary, we compute that

$$
\begin{aligned}
& \partial_{t} F\left(u_{k}, v_{k}\right) \\
= & -\left(\nabla u_{k}-\chi \frac{u_{k}}{v_{k}} \nabla v_{k}\right)\left(\partial_{u u} F\left(u_{k}, v_{k}\right) \nabla u_{k}+\partial_{u v} F\left(u_{k}, v_{k}\right) \nabla v_{k}\right)+\nabla \cdot\left(\partial_{u} F\left(u_{k}, v_{k}\right)\left(\nabla u_{k}-\chi \frac{u_{k}}{v_{k}} \nabla v_{k}\right)\right) \\
& -\nabla v_{k} \cdot\left(\partial_{u v} F\left(u_{k}, v_{k}\right) \nabla u_{k}+\partial_{v v} F\left(u_{k}, v_{k}\right) \nabla v_{k}\right)+\nabla \cdot\left(\partial_{v} F\left(u_{k}, v_{k}\right) \nabla v_{k}\right) \\
& +\partial_{v} F\left(u_{k}, v_{k}\right)\left(-v_{k}+\frac{u_{k}}{1+\frac{1}{k} u_{k}}\right) \\
=- & \left(\partial_{u u} F\left(u_{k}, v_{k}\right)\left|\nabla u_{k}\right|^{2}+2\left(\partial_{u v} F\left(u_{k}, v_{k}\right)-\frac{\chi}{2} \frac{u_{k}}{v_{k}} \partial_{u u} F\left(u_{k}, v_{k}\right)\right) \nabla u_{k} \cdot \nabla v_{k}\right. \\
& \left.+\left(\partial_{v v} F\left(u_{k}, v_{k}\right)-\chi \frac{u_{k}}{v_{k}} \partial_{u v} F\left(u_{k}, v_{k}\right)\right)\left|\nabla v_{k}\right|^{2}\right) \\
+ & \nabla \cdot\left(\partial_{u} F\left(u_{k}, v_{k}\right) \nabla u_{k}+\left(\partial_{v} F\left(u_{k}, v_{k}\right)-\chi \frac{u_{k}}{v_{k}} \partial_{u} F\left(u_{k}, v_{k}\right)\right) \nabla v_{k}\right)+\partial_{v} F\left(u_{k}, v_{k}\right)\left(-v_{k}+\frac{u_{k}}{1+\frac{1}{k} u_{k}}\right) .
\end{aligned}
$$

Now we choose $F$ as in (1.4). Using the chain rule where necessary, one easily verifies that

$$
\begin{aligned}
& \nabla u_{k}=-\frac{2}{a} u_{k}^{\frac{a}{2}+1} v_{k}^{\frac{b}{2}}\left(\nabla\left(u_{k}^{-\frac{a}{2}} v_{k}^{-\frac{b}{2}}\right)+\frac{b}{2}\left(u_{k}^{-\frac{a}{2}} v_{k}^{-\frac{b}{2}-1} \nabla v_{k}\right)\right) \\
& \nabla v_{k}=u_{k}^{\frac{a}{2}} v_{k}^{\frac{b}{2}+1}\left(u_{k}^{-\frac{a}{2}} v_{k}^{-\frac{b}{2}-1} \nabla v_{k}\right) .
\end{aligned}
$$


Plugging (3.14)-(3.15) into (3.13) we arrive, after some computation, at the following identity:

$$
\begin{aligned}
\partial_{t}\left(u_{k}^{-a} v_{k}^{-b}\right)= & -4\left(\frac{a+1}{a}\left|\nabla\left(u_{k}^{-\frac{a}{2}} v_{k}^{-\frac{b}{2}}\right)\right|^{2}+\left(\frac{b}{a}+\chi \frac{a+1}{2}\right) \nabla\left(u_{k}^{-\frac{a}{2}} v_{k}^{-\frac{b}{2}}\right) \cdot u_{k}^{-\frac{a}{2}} v_{k}^{-\frac{b}{2}-1} \nabla v_{k}\right. \\
& \left.+\frac{1}{4}\left(\frac{b^{2}}{a}+b+\chi b\right)\left|u_{k}^{-\frac{a}{2}} v_{k}^{-\frac{b}{2}-1} \nabla v_{k}\right|^{2}\right) \\
& +\nabla \cdot\left(\nabla\left(u_{k}^{-a} v_{k}^{-b}\right)+\chi a u_{k}^{-a} v_{k}^{-b-1} \nabla v_{k}\right)+b u_{k}^{-a} v_{k}^{-b}-b \frac{u_{k}^{-a+1}}{1+\frac{1}{k} u_{k}} v_{k}^{-b-1} .
\end{aligned}
$$

Multiplying (3.16) by an arbitrary function $\psi \in C_{0}^{1}\left(\mathbb{R}_{0}^{+}\right)$and integrating by parts w.r.t. $t$ yields for all $x \in \Omega$ that

$$
\begin{aligned}
& -\int_{0}^{\infty} u_{k}^{-a} v_{k}^{-b} \partial_{t} \psi d t-u_{k 0}^{-a} v_{k 0}^{-b} \psi(0) \\
& =-4 \int_{0}^{\infty}\left(\frac{a+1}{a}\left|\nabla\left(u_{k}^{-\frac{a}{2}} v_{k}^{-\frac{b}{2}}\right)\right|^{2}+\left(\frac{b}{a}+\chi \frac{a+1}{2}\right) \nabla\left(u_{k}^{-\frac{a}{2}} v_{k}^{-\frac{b}{2}}\right) \cdot u_{k}^{-\frac{a}{2}} v_{k}^{-\frac{b}{2}-1} \nabla v_{k}\right. \\
& \left.\quad+\frac{1}{4}\left(\frac{b^{2}}{a}+b+\chi b\right)\left|u_{k}^{-\frac{a}{2}} v_{k}^{-\frac{b}{2}-1} \nabla v_{k}\right|^{2}\right) \psi d t \\
& +\nabla \cdot \int_{0}^{\infty}\left(\nabla\left(u_{k}^{-a} v_{k}^{-b}\right)+\chi a u_{k}^{-a} v_{k}^{-b-1} \nabla v_{k}\right) \psi d t+\int_{0}^{\infty}\left(b u_{k}^{-a} v_{k}^{-b}-b \frac{u_{k}^{-a+1}}{1+\frac{1}{k} u_{k}} v_{k}^{-b-1}\right) \psi
\end{aligned}
$$

On the other hand, multiplying (3.16) by an arbitrary function $\varphi \in C^{1}(\bar{\Omega})$ and integrating by parts w.r.t. $x$ and using the boundary conditions where necessary yields that

$$
\begin{aligned}
\int_{\Omega} \partial_{t}\left(u_{k}^{-a} v_{k}^{-b}\right) \varphi= & -4 \int_{\Omega}\left(\frac{a+1}{a}\left|\nabla\left(u_{k}^{-\frac{a}{2}} v_{k}^{-\frac{b}{2}}\right)\right|^{2}+\left(\frac{b}{a}+\chi \frac{a+1}{2}\right) \nabla\left(u_{k}^{-\frac{a}{2}} v_{k}^{-\frac{b}{2}}\right) \cdot u_{k}^{-\frac{a}{2}} v_{k}^{-\frac{b}{2}-1} \nabla v_{k}\right. \\
& \left.+\frac{1}{4}\left(\frac{b^{2}}{a}+b+\chi b\right)\left|u_{k}^{-\frac{a}{2}} v_{k}^{-\frac{b}{2}-1} \nabla v_{k}\right|^{2}\right) \varphi d x \\
& -\int_{\Omega}\left(\nabla\left(u_{k}^{-a} v_{k}^{-b}\right)+\chi a u_{k}^{-a} v_{k}^{-b-1} \nabla v_{k}\right) \cdot \nabla \varphi d x \\
& +\int_{\Omega}\left(b u_{k}^{-a} v_{k}^{-b}-b \frac{u_{k}^{-a+1}}{1+\frac{1}{k} u_{k}} v_{k}^{-b-1}\right) \varphi d x
\end{aligned}
$$

Finally, if we multiply (3.16) by the product $\psi \varphi$ and integrate by parts w.r.t. $t$ and $x$, then we arrive at the following variational reformulation of (3.16):

$$
\begin{aligned}
& -\int_{0}^{\infty} \partial_{t} \psi \int_{\Omega} u_{k}^{-a} v_{k}^{-b} \varphi d x d t-\psi(0) \int_{\Omega} u_{k 0}^{-a} v_{k 0}^{-b} \varphi d x \\
& =-4 \int_{0}^{\infty} \psi \int_{\Omega}\left(\frac{a+1}{a}\left|\nabla\left(u_{k}^{-\frac{a}{2}} v_{k}^{-\frac{b}{2}}\right)\right|^{2}+\left(\frac{b}{a}+\chi \frac{a+1}{2}\right) \nabla\left(u_{k}^{-\frac{a}{2}} v_{k}^{-\frac{b}{2}}\right) \cdot u_{k}^{-\frac{a}{2}} v_{k}^{-\frac{b}{2}-1} \nabla v_{k}\right. \\
& \left.\quad+\frac{1}{4}\left(\frac{b^{2}}{a}+b+\chi b\right)\left|u_{k}^{-\frac{a}{2}} v_{k}^{-\frac{b}{2}-1} \nabla v_{k}\right|^{2}\right) \varphi d x d s \\
& -\int_{0}^{\infty} \psi \int_{\Omega}\left(\nabla\left(u_{k}^{-a} v_{k}^{-b}\right)+\chi a u_{k}^{-a} v_{k}^{-b-1} \nabla v_{k}\right) \cdot \nabla \varphi d x d s \\
& +\int_{0}^{\infty} \psi \int_{\Omega}\left(b u_{k}^{-a} v_{k}^{-b}-b \frac{u_{k}^{-a+1}}{1+\frac{1}{k} u_{k}} v_{k}^{-b-1}\right) \varphi d x d s .
\end{aligned}
$$

For equation (3.1b) a standard procedure yields the following reformulations: for all $0 \leqslant \varphi \in C^{1}(\bar{\Omega})$ and $0 \leqslant \psi \in C_{0}^{1}\left(\mathbb{R}_{0}^{+}\right)$it holds that

$$
-\int_{0}^{\infty} v_{k} \partial_{t} \psi d s-\psi(0) v_{k 0}=\nabla \cdot \int_{0}^{\infty} \psi \nabla v_{k} d t+\int_{0}^{\infty}\left(-v_{k}+\frac{u_{k}}{1+\frac{1}{k} u_{k}}\right) \psi d t
$$

and

$$
-\int_{0}^{\infty} \partial_{t} \psi \int_{\Omega} v_{k} \varphi d x d s-\psi(0) \int_{\Omega} v_{k 0} \varphi d x=\int_{0}^{\infty} \psi \int_{\Omega}-\nabla v_{k} \cdot \nabla \varphi+\left(-v_{k}+\frac{u_{k}}{1+\frac{1}{k} u_{k}}\right) \varphi d x d s .
$$




\subsection{Further uniform estimates for (3.1)}

Choosing $\varphi \equiv 1$ in (3.18) yields that

$$
\begin{aligned}
\frac{d}{d t} \int_{\Omega} u_{k}^{-a} v_{k}^{-b} d x=\int_{\Omega}-4( & \frac{a+1}{a}\left|\nabla\left(u_{k}^{-\frac{a}{2}} v_{k}^{-\frac{b}{2}}\right)\right|^{2}+\left(\frac{b}{a}+\chi \frac{a+1}{2}\right) \nabla\left(u_{k}^{-\frac{a}{2}} v_{k}^{-\frac{b}{2}}\right) \cdot u_{k}^{-\frac{a}{2}} v_{k}^{-\frac{b}{2}-1} \nabla v_{k} \\
& \left.+\frac{1}{4}\left(\frac{b^{2}}{a}+b+\chi b\right)\left|u_{k}^{-\frac{a}{2}} v_{k}^{-\frac{b}{2}-1} \nabla v_{k}\right|^{2}\right)+b u_{k}^{-a} v_{k}^{-b}-b \frac{u_{k}^{-a+1}}{1+\frac{1}{k} u_{k}} v_{k}^{-b-1} d x .
\end{aligned}
$$

Due to the key assumption (1.5) it holds that

$$
\begin{aligned}
& 0>\left(\frac{b}{a}+\chi \frac{a+1}{2}\right)^{2}-\frac{a+1}{a}\left(\frac{b^{2}}{a}+b+\chi b\right)=\chi^{2} \frac{(a+1)^{2}}{4}-\frac{(b+a+1) b}{a} \\
\Leftrightarrow & \chi^{2}<4 \frac{(b+a+1) b}{a(a+1)^{2}} .
\end{aligned}
$$

Thus, the quadratic form

$$
Q(U, V):=4\left(\frac{a+1}{a}|U|^{2}+\left(\frac{b}{a}+\chi \frac{a+1}{2}\right) U \cdot V+\frac{1}{4}\left(\frac{b^{2}}{a}+b+\chi b\right)|V|^{2}\right)
$$

is strictly convex and satisfies

$$
Q(U, V) \geqslant C_{3}\left(|U|^{2}+|V|^{2}\right) \quad \text { for all } U, V \in \mathbb{R}^{n} .
$$

Then, due to (3.25), we have with (3.22) that

$$
\frac{d}{d t} \int_{\Omega} u_{k}^{-a} v_{k}^{-b} d x+\int_{\Omega} C_{3}\left(\left|\nabla\left(u_{k}^{-\frac{a}{2}} v_{k}^{-\frac{b}{2}}\right)\right|^{2}+\left|u_{k}^{-\frac{a}{2}} v_{k}^{-\frac{b}{2}-1} \nabla v_{k}\right|^{2}\right)+b \frac{u_{k}^{-a+1}}{1+\frac{1}{k} u_{k}} v_{k}^{-b-1} d x \leqslant b \int_{\Omega} u_{k}^{-a} v_{k}^{-b} d x
$$

Applying Gronwall's lemma to the differential inequality (3.26) we arrive at

$$
\begin{aligned}
& \int_{\Omega} u_{k}^{-a} v_{k}^{-b} d x+\int_{0}^{t} \int_{\Omega} C_{3}\left(\left|\nabla\left(u_{k}^{-\frac{a}{2}} v_{k}^{-\frac{b}{2}}\right)\right|^{2}+\left|u_{k}^{-\frac{a}{2}} v_{k}^{-\frac{b}{2}-1} \nabla v_{k}\right|^{2}\right)+b \frac{u_{k}^{-a+1}}{1+\frac{1}{k} u_{k}} v_{k}^{-b-1} d x d s \\
\leqslant & e^{b t} \int_{\Omega} u_{k 0}^{-a} v_{k 0}^{-b} d x \\
\leqslant & C_{4}(T) .
\end{aligned}
$$

Integral inequality (3.27) yields the following set of estimates:

$$
\begin{aligned}
& \left\|\nabla\left(u_{k}^{-\frac{a}{2}} v_{k}^{-\frac{b}{2}}\right)\right\|_{L^{2}((0, T) \times \Omega)} \leqslant C_{5}(T), \\
& \left\|u_{k}^{-\frac{a}{2}} v_{k}^{-\frac{b}{2}-1} \nabla v_{k}\right\|_{L^{2}((0, T) \times \Omega)} \leqslant C_{5}(T), \\
& \left\|u_{k}^{-\frac{a}{2}} v_{k}^{-\frac{b}{2}}\right\|_{L^{\infty}\left(0, T ; L^{2}(\Omega)\right)} \leqslant C_{5}(T), \\
& \left\|\frac{u_{k}^{-a+1}}{1+\frac{1}{k} u_{k}} v_{k}^{-b-1}\right\|_{L^{1}((0, T) \times \Omega)} \leqslant C_{5}(T) .
\end{aligned}
$$

Due to a Sobolev-type inequality (see, e.g., [13, Chapter II $§ 3(3.4)]$ ), estimates (3.28) and (3.30) imply that

$$
\left\|u_{k}^{-a} v_{k}^{-b}\right\|_{L^{1+\frac{2}{n}}((0, T) \times \Omega)} \leqslant C_{6}(T) .
$$

Thanks to estimates (3.28) and (3.32) and the Hölder inequality we obtain that

$$
\left\|\nabla\left(u_{k}^{-a} v_{k}^{-b}\right)\right\|_{L^{\frac{n+2}{n+1}}((0, T) \times \Omega)} \leqslant C_{7}(T) .
$$


Similarly, estimates (3.29) and (3.32) and the Hölder inequality imply that

$$
\left\|u_{k}^{-a} v_{k}^{-b-1} \nabla v_{k}\right\|_{L^{\frac{n+2}{n+1}}((0, T) \times \Omega)} \leqslant C_{8}(T) .
$$

Combining (3.33)-(3.34) we conclude that

$$
\left\|\nabla\left(u_{k}^{-a} v_{k}^{-b}\right)+\chi a u_{k}^{-a} v_{k}^{-b-1} \nabla v_{k}\right\|_{L^{\frac{n+2}{n+1}}((0, T) \times \Omega)} \leqslant C_{9}(T) .
$$

Thanks to (3.28)-(3.31) and (3.35) we deduce from (3.18) that

$$
\left\|\partial_{t}\left(u_{k}^{-a} v_{k}^{-b}\right)\right\|_{L^{1}\left(0, T ;\left(W^{1, n+2}(\Omega)\right)^{*}\right)} \leqslant C_{10} .
$$

Further, due to (3.5) and (3.28)-(3.31) we deduce from (3.17) that for all $\psi \in C_{0}^{1}\left(\mathbb{R}_{0}^{+}\right)$it holds that

$$
\left\|\nabla \cdot \int_{0}^{\infty} \psi\left(\nabla\left(u_{k}^{-a} v_{k}^{-b}\right)+\chi a u_{k}^{-a} v_{k}^{-b-1} \nabla v_{k}\right) d t\right\|_{L^{1}(\Omega)} \leqslant C_{11}(\psi) .
$$

Similarly, using (3.4) and (3.9)-(3.10) we deduce from (3.20) that

$$
\left\|\nabla \cdot \int_{0}^{\infty} \psi \nabla v_{k} d t\right\|_{L^{1}(\Omega)} \leqslant C_{12}(\psi) .
$$

Remark 3.1 (Sign of b). Condition (3.23) would also be satisfied if

$$
b<b_{-}(a):=-\frac{1+a}{2}\left(\sqrt{1+\chi^{2} a}+1\right) .
$$

However, in this case

$$
b<0,
$$

which implies that the sign of the last term on the left-hand side of (3.26) is positive. Consequently, the immediate control upon this term is lost. Similar to the analysis [15] one would then be forced to impose restrictions upon $\chi$ and the space dimension $n$ in order to be able to bind it by means of other terms.

\section{Convergence to a generalised supersolution of (1.1): proof of} Theorem 2.6

Throughout this sequel we assume that

$$
0 \leqslant \varphi \in C^{1}(\bar{\Omega}), \quad 0 \leqslant \psi \in C_{0}^{1}\left(\mathbb{R}_{0}^{+}\right),
$$

and are arbitrary.

Based on the properties established in Section 3 we conclude that a subsequence $k_{m}$ and measurable functions $u, v, \eta:[0, \infty) \times \bar{\Omega} \rightarrow \mathbb{R}^{+}$exist, such that:

due to (3.11),

$$
\begin{array}{ll}
v_{k_{m}} \underset{m \rightarrow \infty}{\rightarrow} v & \text { in } L_{l o c}^{r}\left(\mathbb{R}_{0}^{+} \times \bar{\Omega}\right) \text { and a.e. in } \mathbb{R}^{+} \times \Omega, \\
\nabla v_{k_{m}} \underset{m \rightarrow \infty}{\rightarrow} \nabla v & \text { in } L_{l o c}^{s}\left(\mathbb{R}_{0}^{+} \times \bar{\Omega}\right) \text { and a.e. in } \mathbb{R}^{+} \times \Omega ;
\end{array}
$$

due to (3.12) and (4.1), for all $0<\tau<T<\infty$

$$
\underset{(\tau, T) \times \Omega}{\operatorname{essinf}} v \geqslant C_{2}(\tau, T)>0,
$$

so that $v^{-1} \in L_{l o c}^{\infty}\left(\mathbb{R}^{+} \times \bar{\Omega}\right)$;

due to (3.33), (3.36), and the Lions-Aubin lemma [19, Corollary 4],

$$
u_{k_{m}}^{-a} v_{k_{m}}^{-b} \underset{m \rightarrow \infty}{\rightarrow} \eta \quad \text { in } L_{l o c}^{1}\left(\mathbb{R}_{0}^{+} \times \bar{\Omega}\right) \text { and a.e. in } \mathbb{R}^{+} \times \Omega ;
$$


due to (4.1) and (4.4),

$$
\begin{aligned}
& u_{k_{m}}^{-1}=\left(u_{k_{m}}^{-a} v_{k_{m}}^{-b}\right)^{\frac{1}{a}} v_{k_{m}}^{\frac{b}{a}} \\
& \underset{m \rightarrow \infty}{\rightarrow} \eta^{\frac{1}{a}} v^{\frac{b}{a}} \in[0, \infty) \quad \text { a.e. in } \mathbb{R}^{+} \times \Omega ;
\end{aligned}
$$

due to (4.5),

$$
u_{k_{m}} \underset{m \rightarrow \infty}{\rightarrow} \eta^{-\frac{1}{a}} v^{-\frac{b}{a}}=: u \in(0, \infty] \quad \text { a.e. in } \mathbb{R}^{+} \times \Omega ;
$$

due to (3.9), (4.6), and Fatou's lemma,

$$
u_{k_{m}} \underset{m \rightarrow \infty}{\rightarrow} u \in(0, \infty) \quad \text { a.e. in } \mathbb{R}^{+} \times \Omega,
$$

and $u \in L^{\infty}\left(\mathbb{R}^{+} ; L^{1}(\Omega)\right)$;

due to (4.1), (4.4), (4.7),

$$
u_{k_{m}}^{-a} v_{k_{m}}^{-b} \underset{m \rightarrow \infty}{\rightarrow} u^{-a} v^{-b} \quad \text { in } L_{l o c}^{1}\left(\mathbb{R}_{0}^{+} \times \bar{\Omega}\right) \text { and a.e. in } \mathbb{R}^{+} \times \Omega ;
$$

due to (3.29), (4.1), (4.2), (4.7), and the Lions lemma,

$$
u_{k_{m}}^{-\frac{a}{2}} v_{k_{m}}^{-\frac{b}{2}-1} \nabla v_{k_{m}} \underset{m \rightarrow \infty}{\rightarrow} u^{-\frac{a}{2}} v^{-\frac{b}{2}-1} \nabla v \quad \text { in } L_{l o c}^{2}\left(\mathbb{R}_{0}^{+} \times \bar{\Omega}\right) ;
$$

due to (3.28), (4.8), and the Banach-Alaoglu theorem,

$$
\nabla\left(u_{k_{m}}^{-\frac{a}{2}} v_{k_{m}}^{-\frac{b}{2}}\right) \underset{m \rightarrow \infty}{\rightarrow} \nabla\left(u^{-\frac{a}{2}} v^{-\frac{b}{2}}\right) \quad \text { in } L_{l o c}^{2}\left(\mathbb{R}_{0}^{+} \times \bar{\Omega}\right) ;
$$

due to (3.33), (4.8), and the Banach-Alaoglu theorem,

$$
\nabla\left(u_{k_{m}}^{-a} v_{k_{m}}^{-b}\right) \underset{m \rightarrow \infty}{\rightarrow} \nabla\left(u^{-a} v^{-b}\right) \quad \text { in } L_{l o c}^{\frac{n+2}{n+1}}\left(\mathbb{R}_{0}^{+} \times \bar{\Omega}\right) ;
$$

due to (3.34), (4.1), (4.2), (4.7), and the Lions lemma,

$$
u_{k_{m}}^{-a} v_{k_{m}}^{-b-1} \nabla v_{k_{m}} \underset{m \rightarrow \infty}{\rightarrow} u^{-a} v^{-b-1} \nabla v \quad \text { in } L_{l o c}^{\frac{n+2}{n+1}}\left(\mathbb{R}_{0}^{+} \times \bar{\Omega}\right) ;
$$

due to (4.11)-(4.12),

$$
\nabla\left(u_{k_{m}}^{-a} v_{k_{m}}^{-b}\right)+u_{k_{m}}^{-a} v_{k_{m}}^{-b-1} \nabla v_{k_{m}} \underset{m \rightarrow \infty}{\rightarrow} \nabla\left(u^{-a} v^{-b}\right)+u^{-a} v^{-b-1} \nabla v \quad \text { in } L_{l o c}^{\frac{n+2}{n+1}}\left(\mathbb{R}_{0}^{+} \times \bar{\Omega}\right) ;
$$

due to (4.1) and (4.7),

$$
\frac{u_{k_{m}}^{-a+1}}{1+\frac{1}{k_{m}} u_{k_{m}}} v_{k_{m}}^{-b-1} \underset{m \rightarrow \infty}{\rightarrow} u^{-a+1} v^{-b-1} \quad \text { a.e. in } \mathbb{R}^{+} \times \Omega
$$

due to (3.31), (4.14), and Fatou's lemma,

$$
\liminf _{m \rightarrow \infty} \int_{0}^{\infty} \psi \int_{\Omega} \frac{u_{k_{m}}^{-a+1}}{1+\frac{1}{k_{m}} u_{k_{m}}} v_{k_{m}}^{-b-1} \varphi d x d s \geqslant \int_{0}^{\infty} \psi \int_{\Omega} u^{-a+1} v^{-b-1} \varphi d x d s,
$$

and $u^{-a+1} v^{-b-1} \in L_{l o c}^{1}\left(\mathbb{R}_{0}^{+} ; L^{1}(\Omega)\right)$;

due to (4.7),

$$
\frac{u_{k_{m}}}{1+\frac{1}{k_{m}} u_{k_{m}}} \underset{m \rightarrow \infty}{\rightarrow} u \quad \text { a.e. in }(0, T) \times \Omega ;
$$

due to (3.3), (3.9), and the Banach-Alaoglu theorem, there exists some $\mu \in \mathcal{M}\left(\mathbb{R}_{0}^{+} \times \bar{\Omega}\right)$ s.t.

$$
\lim _{m \rightarrow \infty} \int_{0}^{\infty} \psi \int_{\Omega} \frac{u_{k_{m}}}{1+\frac{1}{k_{m}} u_{k_{m}}} \varphi d x d s=\int_{0}^{\infty} \psi \int_{\Omega} u \varphi d x d s+\int_{0}^{\infty} \psi \int_{\Omega} \varphi d \mu(t, x)
$$


due to (4.16) and Fatou's lemma,

$$
\lim _{m \rightarrow \infty} \int_{0}^{\infty} \psi \int_{\Omega} \frac{u_{k_{m}}}{1+\frac{1}{k_{m}} u_{k_{m}}} \varphi d x d s \geqslant \int_{0}^{\infty} \psi \int_{\Omega} u \varphi d x d s ;
$$

due to (4.17) and (4.18),

$$
\int_{0}^{\infty} \psi \int_{\bar{\Omega}} \varphi d \mu(t, x) \geqslant 0
$$

so that $\mu$ is a non-negative measure;

due to (3.3) and (3.9),

$$
\begin{aligned}
\int_{0}^{\infty} \psi \int_{\Omega} \frac{u_{k_{m}}}{1+\frac{1}{k_{m}} u_{k_{m}}} d x d s & \leqslant \int_{0}^{\infty} \psi \int_{\Omega} u_{k_{m}} d x d s \\
& =\int_{0}^{\infty} \psi d s \int_{\Omega} u_{k_{m} 0} d x \\
& \rightarrow \int_{m \rightarrow \infty}^{\infty} \psi d s \int_{\Omega} u_{0} d x
\end{aligned}
$$

due to (4.17) (set $\varphi \equiv 1)$ and (4.19), $u$ satisfies (2.5);

due to (4.9), (4.10), and the convexity of $Q$ (was defined in (3.24)), for all $0 \leqslant \varphi \in C([0, T] \times \bar{\Omega}$ )

$$
\begin{gathered}
\liminf _{m \rightarrow \infty} \int_{0}^{T} \int_{\Omega} \varphi Q\left(\nabla\left(u_{k_{m}}^{-\frac{a}{2}} v_{k_{m}}^{-\frac{b}{2}}\right), u_{k_{m}}^{-\frac{a}{2}} v_{k_{m}}^{-\frac{b}{2}-1} \nabla v_{k_{m}}\right) d x d s \\
\geqslant \int_{0}^{T} \int_{\Omega} \varphi Q\left(\nabla\left(u^{-\frac{a}{2}} v^{-\frac{b}{2}}\right), u^{-\frac{a}{2}} v^{-\frac{b}{2}-1} \nabla v\right) d x d s
\end{gathered}
$$

due to (3.37), (4.13), and the Banach-Alaoglu theorem,

$$
\begin{gathered}
\nabla \cdot \int_{0}^{\infty} \psi\left(\nabla\left(u_{k_{m}}^{-a} v_{k_{m}}^{-b}\right)+\chi a u_{k_{m}}^{-a} v_{k_{m}}^{-b-1} \nabla v_{k_{m}}\right) d t \\
\underset{m \rightarrow \infty}{\stackrel{*}{*}} \nabla \cdot \int_{0}^{\infty} \psi\left(\nabla\left(u^{-a} v^{-b}\right)+\chi a u^{-a} v^{-b-1} \nabla v\right) d t \quad \text { in } \mathcal{M}(\bar{\Omega}),
\end{gathered}
$$

and

$$
\int_{0}^{\infty} \psi\left(\nabla\left(u^{-a} v^{-b}\right)+\chi a u^{-a} v^{-b-1} \nabla v\right) d t \in \mathcal{D} \mathcal{M}^{\frac{n+2}{n+1}}(\Omega)
$$

due to (A.2), (3.1c), (4.13), and (4.21), $(u, v)$ satisfies (2.6);

due to (3.38), (4.2), and the Banach-Alaoglu theorem,

$$
\nabla \cdot \int_{0}^{\infty} \psi \nabla v_{k_{m}} d t \underset{m \rightarrow \infty}{\stackrel{*}{\rightarrow}} \nabla \cdot \int_{0}^{\infty} \psi \nabla v d t \quad \text { in } \mathcal{M}(\bar{\Omega}),
$$

and

$$
\int_{0}^{\infty} \psi \nabla v d t \in \mathcal{D M}^{s}(\Omega)
$$

due to (A.2), (3.1c), (4.2), and (4.22), $v$ satisfies (2.7).

Altogether, combining (3.5), (4.8), (4.15), (4.20), and (4.21), we deduce from (3.19) for $k=k_{m}$ by taking limit superior as $m \rightarrow \infty$ that $(u, v)$ satisfies (2.3). Finally, passing to the limit in (3.21) for $k=k_{m}$ as $m \rightarrow \infty$, and using (4.1), (4.2), and (4.17), we find that $(u, v)$ satisfies (2.4). Theorem 2.6 is proved.

\section{Classical solutions to (1.1): proof of Theorem 2.7}

In this final section we prove Theorem 2.7. Thus, we assume now that $u$ and $v$ are smooth and satisfy $(2.9)$ and verify that in this case $(u, v)$ is, in fact, a classical solution to (1.1). 
Since $v$ is smooth, the weak boundary condition $(2.7)$ implies that $\partial_{\nu} v(t, \cdot)$ vanishes on the boundary of $\partial \Omega$ for all $t>0$. Further, since both $u$ and $v$ are smooth, the weak boundary condition (2.6) takes the form

$$
\partial_{\nu}\left(u^{-a} v^{-b}\right)+\chi a u^{-a} v^{-b-1} \partial_{\nu} v=0 \quad \text { for all }(t, x) \in(0, T) \times \partial \Omega .
$$

This means that

$$
-a u^{-a-1} v^{-b}\left(\partial_{\nu} u-\chi \frac{u}{v} \partial_{\nu} v\right)-b u^{-a} v^{-b-1} \partial_{\nu} v=0 \quad \text { for all }(t, x) \in(0, T) \times \partial \Omega .
$$

Dividing (5.1) by $-a u^{-a-1} v^{-b}$ and plugging the boundary condition for $v$, we conclude that $\partial_{\nu} u(t, \cdot)$ vanishes on $\partial \Omega$ for all $t>0$ as well.

Next, exploiting the smoothness of $u$ and $v$, we integrate by parts in (2.3) w.r.t. $t$ and $x$ and then apply the Du Bois-Reymond lemma. This results in the differential inequality

$$
\left(-a u^{-a-1} v^{-b}\left(\partial_{t} u-\nabla \cdot\left(\nabla u-\chi \frac{u}{v} \nabla v\right)\right)\right)-b u^{-a} v^{-b-1}\left(\partial_{t} v-(\Delta v-v+u)\right) \leqslant 0 \quad \text { in } \mathbb{R}^{+} \times \Omega .
$$

Similarly, (2.4) implies that $\mu=\xi d x d t$ for some smooth density function $\xi \geqslant 0$ and

$$
\partial_{t} v=\Delta v-v+u+\xi \quad \text { in } \mathbb{R}^{+} \times \Omega .
$$

Dividing (5.2) by $-a u^{-a-1} v^{-b}$ and making use of (5.3) we deduce that

$$
\partial_{t} u-\nabla \cdot\left(\nabla u-\chi \frac{u}{v} \nabla v\right) \geqslant-\frac{b}{a} \frac{u}{v} \xi \quad \text { in } \mathbb{R}^{+} \times \Omega
$$

Integrating (5.4) by parts over $\Omega$ thereby using the boundary conditions and, subsequently, integrating over $(0, t)$ for any $t>0$ we then have that

$$
\|u(t, \cdot)\|_{L^{1}(\Omega)} \geqslant\left\|u_{0}\right\|_{L^{1}(\Omega)}-\frac{b}{a} \int_{0}^{t} \int_{\Omega} \frac{u}{v} \xi d x d s .
$$

On the other hand, due to the Du Bois-Reymond lemma inequality (2.5) is equivalent to the following:

$$
\|u(t, \cdot)\|_{L^{1}(\Omega)}+\|\xi(t, \cdot)\|_{L^{1}(\Omega)} \leqslant\left\|u_{0}\right\|_{L^{1}(\Omega)} \quad \text { for all } t \geqslant 0 .
$$

Thus, combining (5.5)-(5.6) with (2.9) we conclude that

$$
\begin{aligned}
\|\xi(t, \cdot)\|_{L^{1}(\Omega)} & \leqslant \frac{b}{a} \int_{0}^{t} \int_{\Omega} \frac{u}{v} \xi d x d s \\
& \leqslant C_{13}(T) \int_{0}^{t}\|\xi(s, \cdot)\|_{L^{1}(\Omega)} d s \quad \text { for all } 0 \leqslant t \leqslant T<\infty
\end{aligned}
$$

which yields that

$$
\xi \equiv 0
$$

due to the Gronwall lemma. Plugging (5.7) into (5.3), (5.4), and (5.6) immediately yields that (1.1b) is satisfied in the classical sense, and it holds that

$$
\partial_{t} u-\nabla \cdot\left(\nabla u-\chi \frac{u}{v} \nabla v\right) \geqslant 0 \quad \text { in } \mathbb{R}^{+} \times \Omega
$$

and

$$
\|u(t, \cdot)\|_{L^{1}(\Omega)}=\left\|u_{0}\right\|_{L^{1}(\Omega)} \quad \text { for all } t \geqslant 0 .
$$

Since (5.8) is subject to the no-flux boundary conditions, (5.8) and (5.9) imply that equality holds in (5.8). Thus, equation (1.1a) is satisfied in the classical sense, and Theorem 2.7 is proved. 


\section{Appendix A Divergence-measure fields and their normal traces}

In this section we collect some facts concerning the divergence-measure fields.

Let $\mathcal{M}(\bar{\Omega})$ denote the space of Radon measures in $\bar{\Omega}$. We recall the definition of the Banach space of divergence-measure fields [5] and its norm:

$$
\begin{aligned}
& \mathcal{D} \mathcal{M}^{p}(\Omega):=\left\{F \in\left(L^{p}(\Omega)\right)^{n} \mid \nabla \cdot F \in \mathcal{M}(\bar{\Omega})\right\}, \\
& \|F\|_{\mathcal{D} \mathcal{M}^{p}(\Omega)}:=\|F\|_{\left(L^{p}(\Omega)\right)^{n}}+\|\nabla \cdot F\|_{\mathcal{M}(\bar{\Omega})} .
\end{aligned}
$$

Thereby we assume that $p \in\left(1, \frac{n}{n-1}\right)$, which is sufficient for our needs. Following [5] we introduce a generalisation of the normal trace over the boundary of $\partial \Omega$ which automatically satisfies a Gauss-Green formula:

$$
\left\langle\left. F \cdot \nu\right|_{\partial \Omega}, \varphi\right\rangle:=\int_{\Omega} F \cdot \nabla(\mathcal{E} \varphi) d x+\int_{\bar{\Omega}}(\mathcal{E} \varphi) d(\nabla \cdot F) \quad \text { for all } \varphi \in W^{\frac{1}{p}, \frac{p}{p-1}}(\partial \Omega) .
$$

Here $\mathcal{E}: W^{\frac{1}{p}, \frac{p}{p-1}}(\partial \Omega) \rightarrow W^{1, \frac{p}{p-1}}(\Omega)$ is a usual extension operator, i.e., a continuous right inverse of the corresponding trace operator. It is known (see [5, Theorem 2.1]) that $\left.F \cdot \nu\right|_{\partial \Omega} \in W^{-\frac{1}{p}, p}(\partial \Omega)$ and doesn't depend upon the particular choice of $\mathcal{E}$. Formula (A.1) ensures the following implication:

$$
\left\{\left.\left.\begin{array}{ll}
F_{m} \underset{m \rightarrow \infty}{\rightarrow} F & \text { in }\left(L^{p}(\Omega)\right)^{n}, \\
\nabla \cdot F_{m} \underset{m \rightarrow \infty}{\stackrel{*}{*}} \nabla \cdot F & \text { in } \mathcal{M}(\bar{\Omega})
\end{array} \quad \Rightarrow \quad F_{m} \cdot \nu\right|_{\partial \Omega} \underset{m \rightarrow \infty}{\stackrel{*}{*}} F \cdot \nu\right|_{\partial \Omega} \quad \text { in } W^{-\frac{1}{p}, p}(\partial \Omega) .\right.
$$

\section{References}

[1] H. Amann. "Nonhomogeneous linear and quasilinear elliptic and parabolic boundary value problems." In: Function spaces, differential operators and nonlinear analysis. Survey articles and communications of the international conference held in Friedrichsroda, Germany, September 20-26, 1992. Stuttgart: B. G. Teubner Verlagsgesellschaft, 1993, pp. 9-126.

[2] N. Bellomo, A. Bellouquid, Y. Tao, and M. Winkler. "Toward a mathematical theory of Keller-Segel models of pattern formation in biological tissues". In: Math. Models Methods Appl. Sci. 25.9 (2015), pp. 1663-1763. URL: http://dx.doi.org/10.1142/S021820251550044X.

[3] T. Black. Global generalized solutions to a parabolic-elliptic Keller-Segel system with singular sensitivity. preprint. URL: https://arxiv.org/abs/1705.06445.

[4] D. Bothe and M. Pierre. "Quasi-steady-state approximation for a reaction-diffusion system with fast intermediate". In: Journal of Mathematical Analysis and Applications 368.1 (2010), pp. 120 -132. URL: http://www.sciencedirect.com/science/article/pii/S0022247X10001794.

[5] G.-Q. Chen and H. Frid. "On the theory of divergence-measure fields and its applications". In: Bol. Soc. Brasil. Mat. (N.S.) 32.3 (2001). Dedicated to Constantine Dafermos on his 60th birthday, pp. 401-433. URL: https://doi.org/10.1007/BF01233674.

[6] K. Fujie. "Boundedness in a fully parabolic chemotaxis system with singular sensitivity". In: $J$. Math. Anal. Appl. 424.1 (2015), pp. 675-684. uRL: https://doi.org/10.1016/j.jmaa.2014.11. 045.

[7] K. Fujie and T. Senba. "A sufficient condition of sensitivity functions for boundedness of solutions to a parabolic-parabolic chemotaxis system". In: Nonlinearity 31.4 (2018), p. 1639. URL: http: //stacks. iop.org/0951-7715/31/i=4/a=1639.

[8] K. Fujie and T. Senba. "Global existence and boundedness in a parabolic-elliptic Keller-Segel system with general sensitivity". In: Discrete Contin. Dyn. Syst. Ser. B 21.1 (2016), pp. 81-102. URL: https://doi.org/10.3934/dcdsb.2016.21.81.

[9] K. Fujie and T. Senba. "Global existence and boundedness of radial solutions to a two dimensional fully parabolic chemotaxis system with general sensitivity". In: Nonlinearity 29.8 (2016), pp. 24172450. URL: https://doi.org/10.1088/0951-7715/29/8/2417.

[10] D. Horstmann. "From 1970 until present: the Keller-Segel model in chemotaxis and its consequences. I". In: Jahresber. Deutsch. Math.-Verein. 105.3 (2003), pp. 103-165. 
[11] E. F. Keller and L. A. Segel. "Initiation of slime mold aggregation viewed as an instability". In: Journal of Theoretical Biology 26.3 (1970), pp. 399 -415. URL: http://www.sciencedirect.com/ science/article/pii/0022519370900925.

[12] E. F. Keller and L. A. Segel. "Model for chemotaxis". In: Journal of Theoretical Biology 30.2 (1971), pp. 225 -234. URL: http://www. sciencedirect.com/science/article/pii/0022519371900506.

[13] O. Ladyzhenskaya, V. Solonnikov, and N. Ural'tseva. Linear and quasi-linear equations of parabolic type. Translated from the Russian by S. Smith. Translations of Mathematical Monographs. 23. Providence, RI: American Mathematical Society (AMS). XI, 648 p. (1968). 1968.

[14] J. Lankeit. "A new approach toward boundedness in a two-dimensional parabolic chemotaxis system with singular sensitivity". In: Math. Methods Appl. Sci. 39.3 (2016), pp. 394-404. URL: https: //doi.org/10.1002/mma.3489.

[15] J. Lankeit and M. Winkler. "A generalized solution concept for the Keller-Segel system with logarithmic sensitivity: global solvability for large nonradial data". In: Nonlinear Differential Equations and Applications NoDEA 24.4 (2017), p. 49. URL: https://doi.org/10.1007/s00030-017-04728.

[16] S. Luckhaus, Y. Sugiyama, and J. J. L. Velázquez. "Measure valued solutions of the 2D Keller-Segel system". In: Arch. Ration. Mech. Anal. 206.1 (2012), pp. 31-80. URL: https://doi.org/10.1007/ s00205-012-0549-9.

[17] M. Mizukami and T. Yokota. "A unified method for boundedness in fully parabolic chemotaxis systems with signal-dependent sensitivity". In: Math. Nachr. 290.16 (2017), pp. 2648-2660. URL: https://doi.org/10.1002/mana.201600399.

[18] T. Nagai and T. Senba. "Global existence and blow-up of radial solutions to a parabolic-elliptic system of chemotaxis". In: Adv. Math. Sci. Appl. 8.1 (1998), pp. 145-156.

[19] J. Simon. "Compact sets in the space $L^{p}(0, T ; B)$." In: Ann. Mat. Pura Appl. (4) 146 (1987), pp. 65-96.

[20] C. Stinner and M. Winkler. "Global weak solutions in a chemotaxis system with large singular sensitivity". In: Nonlinear Anal. Real World Appl. 12.6 (2011), pp. 3727-3740. URL: https://doi. org/10.1016/j.nonrwa.2011.07.006.

[21] J. I. Tello and M. Winkler. "Reduction of critical mass in a chemotaxis system by external application of a chemoattractant". In: Ann. Sc. Norm. Super. Pisa Cl. Sci. (5) 12.4 (2013), pp. 833862 .

[22] M. Winkler. "Global solutions in a fully parabolic chemotaxis system with singular sensitivity". In: Math. Methods Appl. Sci. 34.2 (2011), pp. 176-190. URL: https://doi.org/10.1002/mma.1346.

[23] M. Winkler. "Large-data global generalized solutions in a chemotaxis system with tensor-valued sensitivities". In: SIAM J. Math. Anal. 47.4 (2015), pp. 3092-3115. URL: https://doi .org/10. 1137/140979708.

[24] M. Winkler. "The two-dimensional Keller-Segel system with singular sensitivity and signal absorption: global large-data solutions and their relaxation properties". In: Math. Models Methods Appl. Sci. 26.5 (2016), pp. 987-1024. URL: https://doi.org/10.1142/S0218202516500238.

[25] X. Zhao and S. Zheng. "Global boundedness of solutions in a parabolic-parabolic chemotaxis system with singular sensitivity". In: J. Math. Anal. Appl. 443.1 (2016), pp. 445-452. URL: https://doi. org/10.1016/j.jmaa.2016.05.036.

[26] A. Zhigun, C. Surulescu, and A. Hunt. "A strongly degenerate diffusion-haptotaxis model of tumour invasion under the go-or-grow dichotomy hypothesis". In: Mathematical Methods in the Applied Sciences 41.6 (2018), pp. 2403-2428. URL: http://dx.doi.org/10.1002/mma. 4749.

[27] A. Zhigun, C. Surulescu, and A. Uatay. "Global existence for a degenerate haptotaxis model of cancer invasion". In: Z. Angew. Math. Phys. 67.6 (2016), p. 146. URL: http://dx.doi .org/10. 1007/s00033-016-0741-0. 